\title{
Les rituels médicaux du sportif
}

Bernard Andrieu

\section{(2) OpenEdition}

Journals

Édition électronique

URL : http://journals.openedition.org/corpsetculture/625

DOI : 10.4000/corpsetculture.625

ISSN : $1777-5337$

\section{Éditeur}

Association Corps et Culture

Édition imprimée

Date de publication : 1 juin 1999

ISSN : 1268-5631

\section{Référence électronique}

Bernard Andrieu, «Les rituels médicaux du sportif », Corps et culture [En ligne], Numéro 4| 1999, mis en ligne le 25 avril 2007, consulté le 08 septembre 2020. URL : http://journals.openedition.org/ corpsetculture/625; DOI : https://doi.org/10.4000/corpsetculture.625

Ce document a été généré automatiquement le 8 septembre 2020

(c) tous droits réservés 


\title{
Les rituels médicaux du sportif
}

\author{
Bernard Andrieu
}

La fin du rite symbolique

1 L'entraînement est un rituel pour le sportif (Rauch A., 1982 : 13-37). Toute sa journée est organisée et économisée en vue de l'entraînement. Sans cette répétition rituelle, l'habituation n'aboutirait pas à l'incorporation. La vertu de l'exercice régulier est de transformer son corps : cet exercice rituel augmente le degré de l'être individuel jusqu'à une limite organique, celle de la résistance et de l'endurance de la structure corporelle aux répétitions successives. La fracture/facture de fatigue vient témoigner jusqu'au martyr de la limite du corps. Le rituel de l'entraînement contient sa limite interne qu'il convient de dépasser. L'initiation reste intime et le dépassement de soi n'isole le sportif qu'en le confrontant avec sa douleur: le rituel sportif engage la diététique et l'hygiène de vie dans une voie médicalisée du sport. Le développement du jogging, entre autres pratiques sportives, développe des médecins du sport (recherches diététique, physiologie de l'effort, pathologie articulaire et tendineuse de la pratique) mais surtout des sportifs-médecins.

2 Car le rituel quotidien du sportif transforme le corps individuel tandis que le rite demeure une insertion du sportif dans une collectivité. Paul Yonnet précise : « ... si l'on observe bien la pratique du jogging une succession de rites de séparation (de la télé, de la voiture, de l'alcool, des femmes), des rites de marge (systèmes d'entraînement à l'écart, souffrances et mutilations physiques, observations d'interdits et de préférences alimentaires) et des rites d'agrégation (dans les épreuves populaires et de "style libre" où le jogger se retrouve à la fois en communauté et en société), si - au terme de ces rites - la naissance d'une nouvelle personnalité suit bien la disparition première, l'espèce de mort décidée de l'ancien individu (le rite d'agrégation célèbre une renaissance, une régénérescence fondée sur une nouvelle hygiène de vie), bref, si l'on est bien là en présence de la séquence des rites et du symbolisme typique de l'initiation, celle-ci n'introduit nullement à des cérémonies marginales, des liturgies élitaires, elle ne rompt jamais avec les données immédiates et essentielles du consensus social» (Yonnet P., 1985 : 115-116). Ainsi l'insertion dans des rites collectifs ne dit rien encore 
de ces rituels si particuliers dans lesquels ces sportifs-médecins entretiennent la mise en culture de leurs corps.

3 Cette mise en culture des corps n'est plus cette éthique de l'acte (Andrieu B., 1994: 211-227) que décrivait Michel Foucault dans les deux derniers tomes de L'Histoire de la sexualité. Le souci de soi ne convoquait pas le sportif grec ou romain au solipsisme: l'individualisme n'avait pas de sens puisque l'entretien physique visait une médecine de l'âme plutôt qu'une maîtrise du corps. La réflexion, qui conduira à une herméneutique $\mathrm{du}$ désir dans le christianisme primitif, devait élaborer l'acte corporel en une esthétique de l'existence. Or aujourd'hui l'esthétique de la violence remplace cette esthétique de l'existence : selon Stamatios Tzizis (Tzizis S., $1997:$ 80-98) la tentation de disposer de son corps comme d'un avoir-droit a engagé l'individu dans une logique de la jouissance. Se faire violence, moins par masochisme, que pour jouir du sport de l'être (Andrieu B., 1998 : 147-168) : l'être ne peut se ressentir que dans la mise en sport de luimême, c'est-à-dire à la fois un épuisement de soi et une incarnation d'un être nouveau. Ce «corporéisme», qu'Yves Le Pogam, analyse comme une croyance en une réappropriation $\mathrm{du}$ corps par une morale des plaisirs, comporte aussi un risque d'aveuglement de l'individu dans les mises en rituel de lui-même (Le Pogam Y., 1997 : 151-169). La "sportification", précise Norbert Elias, instaure le rituel dans l'autodiscipline : l'incorporation de la règle n'est pas suffisante puisque qu'il fallut $\mathrm{y}$ adjoindre une surveillance du respect des règles. Invitant moins au défoulement qu'à la contention, le sport structure l'être dans un rituel régulier.

Dépasser la nature?

4 L'entraînement quotidien transforme le corps jusqu'à la limite biopsychologique du sujet. Sa volonté peut, dès l'effort répété et accumulé, aller au-delà des limites convenues mais le corps ne contient pas la volonté : il peut céder physiquement dans l'effondrement du marathonien ou dans la fringale du cycliste. Le sport devrait être limité par la nature: les capacités naturelles de l'individu définissent une certaine plasticité plutôt qu'un déterminisme inné. L'apprentissage développe cette plasticité en incorporant des états toujours nouveaux. Le sport rejoint ici le projet des sciences biologiques en dépassant la nature par la mise en culture du corps.

5 La performance du record participe donc d'une logique différente de celle de l'entretien de son propre corps. Comme le souligne Yves Travaillot les pratiques d'entretien du corps établissent depuis 1985 une relation moins intime entre forme, santé et beauté au profit d'un hédonisme (Travaillot Y., 1998 : 175-201). Le concept de forme est en perte de vitesse pour être remplacé par l'apologie des médecines douces ou naturelles. Le rituel d'entretien participe d'une économie à l'inverse de la construction artificielle du sportif de haut-niveau. Le paradoxe de notre société est à la fois de mettre en scène la compétition inégalitaire du sport spectacle et de développer le culte individuel du corps. Chacun admet pour lui-même la recherche d'une harmonie naturelle avec son corps tandis que collectivement, il est vrai de plus en plus par les médias interposés, chacun subit aussi l'idéologie combative de corps artificiellement professionnalisés (Moles J.-B., 1998).

6 Ces corps professionnalisés doivent dépasser la nature par la mise en culture de leurs particules élémentaires : aliments, hormones, oxygénation... La relation à la nature est différente selon que le sport vise la santé ou la performance. Les logiques d'entretien physique du corps font de la régularité le principe de l'harmonie naturelle de l'individu avec son corps. L'entretien ne dépasse pas le capital naturel du corps : il le développe 
selon les limites de son économie interne. Au contraire, si le sport moderne est devenu un avatar de la science biologique, c'est en raison de l'amélioration artificielle du corps. L'économie de soi n'est plus seulement narcissique comme dans les années du bodybuilding. Là où la révolution a échoué, l'évolution de l'individu peut désormais réaliser des corps nouveaux.

7 Soigner participe d'une logique curative si bien que le corps social attend de la génétique l'éradication des maladies héréditaires. Le corps, devenu le lieu du sujet, doit correspondre à la norme d'un corps purifié, idéal et esthétique. Ce culte du corps représente à la fois des aspects positifs et négatifs. Du point de vue positif, les progrès de la médecine ont accompagné la libération des mœurs et des attitudes corporelles en révélant combien le sport, la performance physique, le mouvement, la vitesse, la nudité (Urbain J.-D., 1994 ; Kaufmann J.-C., 1995 ; Hennig J.-L., 1995) pouvaient constituer un nouveau mode de problématisation de soi-même face aux autres. La conjugaison de l'individualisme et de l'hédonisme pouvait situer le corps comme le lieu du sujet: la forme, l'apparence, la santé, l'énergie, la durée de vie devinrent des espaces corporels suffisamment consistants pour l'investissement psychosocial des sujets. Chacun se doit d'entretenir sa matière corporelle tout à la fois comme surface sociale, dimension psychologique, image de soi-même. Narcisse trouve désormais son miroir dans le reflet du regard du corps social, regard constitutif de normes esthétiques et de modes symboliques. Du point de vue négatif, cette religion du corps conduit à un certain aveuglement: là où la tradition distinguait l'apparence et l'être, le sujet cherche à localiser toutes les coordonnées de son identité dans des éléments matériels comme le gène, le neurotransmetteur, l'hormone. Si bien qu'une explication naturelle des comportements humains se développe au point d'offrir une réduction du sujet à sa matérialité. La génétique participe de cette dividuation du corps : comme l'indique le philosophe Gilles Deleuze, « on ne se trouve plus devant le couple masse-individu. Les individus sont devenus des "dividuels"... Dans le régime des hôpitaux: la nouvelle médecine » sans médecin ni malade " qui dégage des malades potentiels et des sujets à risque, qui ne témoigne nullement d'un progrès vers l'individuation, comme on le dit, mais substitue au corps individuel ou numérique le chiffre d'une matière "dividuelle" à contrôler » (Deleuze D., 1990 : 244-246). Si l'autogestion de la santé peut être comprise par ses partisans comme le moyen de dessiner une nouvelle carte sanitaire plus rentable, la médecine prédictive place le sujet devant l'économie de son corps: l'entretenir, le faire durer, le gérer comme un capital génétique, éviter de favoriser la réalisation de potentialités malignes en adaptant les usages du corps.

8 La relation à la nature se trouve ainsi profondément modifiée : d'une part le corps individuel peut être grandement et intensément changé dans ses états par l'intervention d'une technologie médicale: par ce moyen l'état du corps dépend de nous pour autant que la démocratie permette l'accès et l'éducation à la santé pour tous. D'autre part la nature même du corps peut être changée par l'intervention scientifique sur les éléments qui constituent l'identité humaine et la singularité de l'ontogenèse individuelle.

La médecine comme rituel de purification

9 La recherche de ces modifications du corps n'est pas seulement une action éphémère. Se modifier est l'action historique de l'individu sur son corps. Ainsi l'individu n'attend plus son salut des sciences de la vie et de la médecine : il le définit en favorisant d'abord la consommation puis en revendiquant de nouveaux services corporels. Sans doute en 
se confiant chaque jour davantage aux médecines dures et douces pourrait-on y voir une instrumentalisation du corps. Rendu dépendant des hormones, des tranquillisants et autres excitants, le sujet est devenu un objet d'expérimentation pharmaceutique. Mais l'habituation aidant, il devient convenu de profiter des avancées des médecines nouvelles pour vivre mieux et plus longtemps : le désir de vivre trouve dans la matière du corps les conditions de sa forme. L'entretien de la forme, mythe des années 80, ne suffit plus: la génétique, l'immunologie et les neurosciences fournissent les mécanismes d'action à tout individu pour dessiner son corps et le corps d'autrui. L'apparence ne suffit plus pour contrôler le regard des autres sur soi. Chacun espère se déterminer en agissant sur les causes de l'apparence.

La sélection humaine d'une population repose sur l'idée de purification de la race. L' héridité suppose une homogénéité dans une lignée afin de purifier les caractères, ce qui est le contraire de la reproduction naturelle toujours hasardeuse et pourvoyeuse de différences. Cette tentation de la pureté est toujours coextensive de la purification ethnique dans la mesure où l'idéologie du racisme justifierait les pratiques de stérilisation et d'élimination. Les fondements de l'eugénisme (Thomas J.-P., 1995) ne datent pas de la génétique puisque l'unité du projet est l'obsession de la décadence. La distinction entre une eugénique positive, animée de la volonté d'améliorer les races humaines en favorisant la fécondité des plus aptes, et d'une eugénique négative, dont l'objet est de restreindre la reproduction des moins aptes continue d'être avancée au cœur même du projet du génome humain: Daniel Cohen n'hésite pas à écrire que «l'accès à la contraception, le droit à l'avortement, comme celui d'avoir des enfants indemnes de maladies graves en ayant recours aux techniques médicales les plus récentes, c'est-à-dire l'eugénisme bien compris, sont une bonne chose, quand ils sont pratiqués bien sûr en toute liberté de choix individuel»(Cohen D., 1993: 229). L'eugénisme négatif, « qui consiste seulement à éviter les naissances d'enfants dont on sait qu'ils seront gravement malades et douloureusement handicapés » (Ibid), ne serait donc pas à craindre puisque la suite logique de l'identification et de la localisation des gènes permettrait d'utiliser le diagnostic génétique pour informer et agir contre la logique naturelle.

11 Même s'il est peu probable de voir la réalisation d'un totalitarisme scientifique, à l'instar du Meilleur des mondes écrit par Huxley en 1932, la force de conviction de l'individualisme démocratique met à la disposition de chacun les moyens techniques d'opérer de manière eugénique. La dérive eugéniste réside dans le passage de la génétique moléculaire à la génétique du comportement dès lors que la naturalisation des fonctions cognitives, par les procédés d'identification et de localisation, entretient l'idée d'un déterminisme plutôt que celle d'une fréquence statistique. Une politique de dépistage, reposant sur l'utilisation systématique des tests, exprime le résurgence du mode de pensée essentialiste en niant la part historique de l'existence humaine. Cette tendance à confondre hérédité avec destinée favorise l'adoption d'une attitude fataliste contre laquelle l'individu, mais surtout le corps social dont il est membre, espère la solution de la recherche génétique. Le passage du fatalisme au dépistage génétique offre en même temps un choix aux individus et une politique de contrôle " général » de la maladie génétique. Le 16 février 1999, le Ministère de l'emploi et de la solidarité révélait des éléments de l'évaluation en cours par la Commission nationale médicale de la biologie, de la reproduction et du diagnostic prénatal : en $199752 \%$ des femmes enceintes ont demandé le dosage des marqueurs sanguins, "évitant " la naissance de 318 enfants trisomiques. Les tests génétiques, effectués sur la base du gène dit de 
l'endurance, ne sont eux aussi que des potentiels statistiques (Morange M., 1998: 181-196) que des entraîneurs pourraient utiliser comme une sélection déterministe des athlètes dès le plus jeune âge.

Cette héridité retrouve les enjeux de la pensée eugénique allemande dont l'État nazi devait réaliser toute l'horreur. S'il devient facile d'associer l'eugénisme au nazisme, il deviendra de plus en plus difficile, suite à la dividuation du corps humain en éléments naturels, de réprimer le désir humain d'une conception économique de l'existence. La médecine génétique peut très rapidement retrouver au nom du bien de ses patients/ clients les solutions eugéniques. Les questions qui et quoi va-t-on soigner? placent le médecin face à de nouvelles responsabilités.

Du placebo au dopage

13 Le placebo est illusion de médicament dont la croyance est subjectivement suffisante pour produire une guérison objective. Cette substance dénuée de principes actifs fait douter la médecine de son efficacité pharmacologique. Prescrit dans un contexte thérapeutique, le placebo est défini par ses effets car rien dans sa cause ne peut faire objectivement attendre une efficacité. Si, précise Patrick Lemoine le placebo a toujours été décrit comme le rêve du médecin magicien, il devient un terme médical en 1785 dans le Motherby's New Medical Dictionnary (Lemoine P., 1996). Le 18 juin 1890 l'éditorial "The placebo in medecine" de Medical Press regrettait la non reconnaissance thérapeutique du placebo. Depuis 1945 la médecine pharmacologique utilise le placebo comme stratégie de validation du médicament ; elle va à l'inverse ainsi de la dimension subjective du placebo en l'utilisant pour faire des essais en aveugle. Ni le patient, ni le médecin, dans l'essai doublement à l'aveugle, ne savent si le traitement contient ou non un placebo. L'information donnée au malade et à sa famille comporte seulement l'obligation d'expliquer le tirage au sort entre un nouveau traitement et un placebo. Si critique que puisse être le placebo, il est utile pour la validation de la pharmacie objective. L'absence de vertu reconnue dans un placebo n'interdit pas son efficacité. D'après la documentation médicale Labaz $\mathrm{n}^{\circ} 11$, les pourcentages d'améliorations subjectives par administration de placebos sont établis ainsi : de 6 à $18 \%$ pour le parkinsonisme, $22 \%$ pour le rhume des foins, $27 \%$ pour la motilité intestinale diminuée, $30 \%$ pour les angoisses et les tremblements, de 4 à $86 \%$ pour les douleurs, de 36 à $43 \%$ pour la toux, de 51 à $60 \%$ d'influence sur la pression sanguine, de 58 à $61 \%$ pour le mal de l'air, de 55 à $88 \%$ pour l'ulcère gastrique et duodénal, et $80 \%$ pour l'arthrite chronique. Ces variations, en fonction des cibles, donnent la mesure de la réceptivité subjective. Les interactions soignants-soignés favorisent plus ou moins l'efficacité du placebo.

Le sportif n'en reste plus au placebo de la volonté pour réaliser absolument une performance. La préparation biologique remplace le volontarisme de l'effort en redéfinissant les conditions de l'évaluation médicale de la performance. Il y donc une différence entre le sport-santé, trois à quatre heures d'activité hebdomadaire, et le sport à haute intensité qui déséquilibre les hormones et développe une traumatologie due directement ou indirectement à la répétition. Le rituel sportif devient alors un processus obsessionnel : enfermement dans le cercle de l'entraînement, développement de soi, isolement socioprofessionnel rendant difficile les reconversions, dépendance $\mathrm{du}$ sportif envers son médecin... En soumettant à un traitement externe, le sportif de hautniveau utilise, rappelle Christian Pociello, "les techniques connues de restauration organique et les procédés scientifiques du "dépassement de soi" " (Pociello C., 1996 : 
28). Cette surnaturation du corps vise la transformation du corps de femmes en corps bisexuel, sinon monohormonal de type masculin, comme l'indique la disparition précoce de Florence Griffith-Joyner et d'autres athlètes d'Allemagne de l'Est. Paul Yonnet ${ }^{1}$ précise : «Le champion est un être doué que l'on doit fatiguer intensément, de façon à obtenir un rendement maximal de la mécanique humaine de cette exploitation consentie d'un capital préexistant, il y aura des gains économiques et symboliques : ce n'est pas un homme dont on cherche à optimiser la santé et le bien être » (Yonnet P., 1998 : 204).

Du soin de soi au soigneur biologique

Le remplacement du médecin de soi-même (Andrieu B., 1999) par le soigneur biologique interdit désormais au sportif de prendre soin de lui-même. Sa dépendance biotechnologique envers le suivi médicalisé transforme la préparation biologique en prise toxicomaniaque. Sans préparation biologique le sportif ne peut rivaliser avec les meilleurs, eux-mêmes sélectionnés sur les mêmes bases pharmacologiques. La résistance aux produits dopants remplace la résistance à l'épreuve sportive. La répétition des compétitions augmente ce seuil de résistance si bien que le vécu corporel du sportif est troublé : la subjectivité du corps vécu évolue au fur et à mesure de la transformation psychobiologique $\mathrm{du}$ corps physique. Cette réorganisation des références subjectives participe de l'habituation toxicomaniaque sans laquelle la réalisation des efforts sera désormais impossible.

Ce détournement des passions, de la nature sacralisée à l'identité revendiquée (Le Pogam Y., 1997 : 33-46) pourrait légitimer le retour de l'homme naturel (Andrieu B., 1999 b). Assainir la société, par le végétarisme, l'homéopathie ou l'aromathérapie (Ouédraogo A.-P., 1998 : 59-76 ; Garetta R., 1998 : 77-88), voudrait l'éradication de toute mise en culture scientifique du corps. Ce retour de l'hygiène transforme le souci du corps (Rauch A., 1983) en une critique du dopage spectacle sans jamais parvenir à remettre en cause le système. Tout le monde fait comme si le spectacle sportif était pur refusant par là même de le consacrer comme un spectacle et en faisant croire en l'authenticité de l'effort. Le changement actuel de règles du jeu de nombreux sports vise l'adaptation aux normes télévisuelles qui rentabilisent l'investissement sportif... cercle vicieux. Admettre que le sport est un spectacle, non seulement dans la scène mais dans les acteurs, reviendrait à dissoudre la limite entre la performance et le cirque. Le professionnalisme, dont le film Rollerball nous indiquait déjà l'objectif politique, ne sert plus seulement de catharsis sociale comme dans les jeux du cirque antique.

Il désigne la limite du corps amateur de sensations pour promouvoir une instrumentalisation du corps (Gleyse J., 1997) dès le plus jeune âge comme le prouve l'organisation psychomotrice des aires de jeux. Rien n'assure que l'éducation psychomotrice aboutisse à une gestion de sa vie physique comme l'espère Jean le Boulch (Le Boulch J., 1998 : 342-382) dans l'école du XXI ${ }^{e}$ siècle; et pas plutôt à une préprofessionnalisation et à une sélection adaptative des meilleurs afin d'assurer les conditions d'un recrutement précoce. Serge Fauché a su dénoncer ces panacées psychomotrices (Fauché S., 1993 : 203-254) qui font du corps éduqué la définition du salut moderne. Cet aveuglement de notre culture dans le corps prive le sport d'être une pratique ludique. En prenant soin de l'énergie biologique du corps, le sportif définit un rituel de dépossession de soi et, en même temps, de redéfinitions de l'être humain. En acceptant de s'en remettre au soigneur, le sportif devient un toxicomane comme les 
autres qui vivent à travers l'alcool, les tranquillisants ou les antalgiques. Comme le rappelle Serge Simon (1998: 7), auteur de l'amendement sur «l'injonction informative " de la loi Buffet votée à l'unanimité, il faut sortir de la logique dopé = tricheur. Les médecins du puissant lobby des commissions médicales des fédérations estiment comme une violation du secret médical, compromettant le contrat de confiance entre le sportif et eux, s'ils devaient déclarer toute suspicion de dopage à la commission médicale attachée au Conseil national de lutte contre le dopage! En maintenant le secret, l'accusation de tricherie est maintenue dans le cadre d'un sport apparemment pur.

Si la société maintient le malade dépendant dans un rituel médical privé c'est, dit-on, pour éviter la réalisation du bouc émissaire et diminuer le contrôle social. En réalité l'économie biologique des pressions et dépressions est bien engagée pour utiliser le corps surnaturé au maximum, en une sorte de cannibalisme médicalisé. La fatigue d'être soi nous rappelle Alain Ehrenberg (Ehrenberg A., 1998 : 250-251) est le miroir tendu à nos normes de socialisation: la ritualisation médicale du corps, du sport au travail (Dejours C., 1998), est moins un contre-pouvoir qu'un pis-aller.

\section{BIBLIOGRAPHIE}

Andrieu B. (1994) Les Cultes du corps. Éthique et sciences, Paris, L'Harmattan.

Andrieu B. (1998) Les Plaisirs de la chair. Une philosophie politique du corps, Paris, Le Temps des cerises.

Andrieu B. (1999) Médecin de son corps, Paris, P.U.F., coll. Médecine et société, Préf. F. Dagognet.

Andrieu B. (1999) L'Homme naturel. De la fin promise des sciences humaines, P.U. Lyon. Préface Guy Avanzini

Cohen D. (1993) Les Gènes de l'espoir. A la découverte du génome humain, Paris, Robert Laffont.

Dejours C. (1998) La Souffrance en France, Paris, Le Seuil.

Deleuze G. (1990) Post-scriptum sur les sociétés de contrôle, Pourparlers, Paris, Minuit.

Ehrenberg A. (1998) La Fatigue d'être soi. Dépression et Société, Paris, O. Jacob.

Fauché S. (1993) Du corps au psychisme. Histoire et épistémologie de la psychomotricité, Paris, P.U.F.

Garreta R. (1998) Ces plantes qui purifient. De l'herboristerie à l'aromathérapie, Terrain, Un corps pur, 31, 77-88.

Gleyse J. (1997) L'Instrumentalisation du corps, Paris, L'Harmattan.

Hennig J.-L. (1995) Brève histoire de fesses, Paris, Calmann-Levy.

Yonnet P. (1985) Jeux, modes et masses 1945-1985. La société française et le moderne, Paris, Gallimard.

Kaufmann J.-C. (1995) Corps de femmes. Regards d'hommes. Sociologie des seins, Paris, Nathan.

Le Boulch J. (1998) Le Corps à l'école au XXIe siècle, Paris, P.U.F. 
Lemoine P. (1996) Le Mystère du placebo, Paris, O. Jacob.

Le Pogam Y. (1997) Corporéisme et individualisme hédoniste, dans Le Pogam Y. (dir), Corps et Culture, Plaisirs du corps, plaisirs du sport, 2, 151-169.

Le Pogam Y. (1997) Passions sportives, identité et modernité, in Quasimodo, Nationalismes sportifs, Printemps 1997, 33-46.

Moles J.-B. (1998) La professionnalisation du rugby français. Pouvoir économique et lien social, in Le Pogam Y. (dir), Corps et Cultures, Sport et lien social, 3, 91-106.

Morange M. (1998) La Part des gènes, Paris, O. Jacob.

Ouédraogo A.P. (1998) Assainir la société. Les enjeux du végétarisme, Terrain, Un corps pur, 31, 59-76.

Pociello C. (1996) Le sport entre mesure et démesure, in Communications, Natures extrêmes, 61, 21-39.

Rauch A. (1982) Le Corps en éducation physique. Histoire et principes de l'entraînement, Paris, P.U.F.

Rauch A. (1983) Le Souci du corps. Histoire de l'hygiène en éducation physique, Paris, P.U.F.

Simon S. (1998) Soigner le dopage, c'est l'accepter, Libération, 8 décembre.

Thomas J.-P. (1995) Les Fondements de l'eugénisme, Paris, P.U.F., coll. Que sais-je, n²2953.

Travaillot Y. (1998) Sociologie des pratiques d'entretien du corps. L'évolution de l'attention portée au corps depuis 1960, Paris, P.U.F.

Urbain J.D. (1994) Sur la plage. Mœurs et coutumes balnéaires, Paris, Payot.

\section{NOTES}

1. Même si Paul Yonnet a pu collaborer avec un journal comme L'Équipe pour faire son étude sur le dopage, l'ambiguïté du journal est d'être à la fois partie prenante (Organisateur) et partie prise (Journaliste) de la course à la performance et de la dénonciation du dopage. $\mathrm{cf}$ " La casse du siècle. Sport et Santé », n 866 , Samedi 14 novembre 1998, Supplément de L'Équipe n¹6.336, p. 42-70.

\section{RÉSUMÉS}

L'entraînement est depuis Aristote la définition du rituel comme acquisition de la vertu. Mais la médicalisation des performances modifie l'exercice en rituel médical : contrôle sanitaire, suivi médical, dopage scientifique. Un sport sans bio-science est-il désormais possible et souhaitable?

Since Aristotle the training is the definition of the ritual as an acquisition of virtue. But the medicalisation of performances change the exercise in medical ritual: health control, medical accompaniment, scientific doping. What is possible a sport without these biosciences? 
INDEX

Keywords : addiction, médicalisation, placebo, doping, medicalisation, virtue Mots-clés : dopage, vertu

\section{AUTEUR}

\section{BERNARD ANDRIEU}

Lycée Louis de Foix de Bayonne. Faculté des Sciences de l'Homme. Université Bordeaux II 